\title{
GAS FLOWS IN S-E BINARY SYSTEMS OF GALAXIES
}

\author{
N. Ya. Sotnikova \\ Astronomical Observatory \\ Leningrad State University \\ Leningrad, USSR
}

\section{OBSERVATIONAL EVIDENCE OF GAS FLOWS IN BINARY SYSTEMS}

Tidal interaction between the galaxies in binary systems leads to important consequences. Some peculiarities in galactic morphology as well as the transfer of matter from one galaxy to another may be due to this factor. In particular, gas flows in intergalactic space may be formed. Such flows enriching one component with gas from the other may play a substantial role in the evolution of mixed (S-E) pairs. One can mention several facts corroborating the possibility of the gas transfer from the spiral to the elliptical galaxy.

1. High HI content $\left(10^{7}-10^{9} \mathrm{M}_{\odot}\right)$ is detected in nearly 40 E galaxies (Bottinelli and Gougenheim, 1979; Knapp et al.,

1985). Such galaxies are often members of pairs or of multiple systems including an S galaxy, which may be the source of gas (Smirnov and Komberg, 1980). Moreover, the gas kinematics and its distribution also indicate an external origin for this gas (Knapp et al., 1985). In many cases there is an outer gaseous disk. The directions of the disk and of stellar rotation don't always coincide (van Gorkom et al., 1985; Varnas et al., 1987). 
2. The galaxy colors in S-E pairs are correlated (the Holmberg effect): bluer ellipticals have spiral components that are usually bluer (Demin et al., 1984).

3. The fraction of $E$ galaxies with emission lines ( $\left.N_{e m}\right)$ in S-E pairs showing traces of tidal interaction is twice as

large $\left(\mathrm{N}_{\mathrm{em}} \simeq 0.24\right)$ as in pairs without interaction $\left(\mathrm{N}_{\mathrm{em}} \simeq 0.12\right)$ (Sotnikova, 1988b). Since the presence of emission lines in a galaxy spectrum strongly depends on gas content this fact also leads to the conclusion that ellipticals in interacting $\mathrm{S}-\mathrm{E}$ pairs are enriched with gas.

These facts may be considered as a serious indication of the existence of gas transfer. Hence, investigation of this process is of interest.

FORM OF THE GASEOUS STREAM

Taking into account the cloud structure of interstellar gas in spiral galaxies, one may assume that the gaseous stream in S-E pairs consists of discrete condensations. Hence, it is possible to consider the motion in the flow within the framework of the restricted three-body problem of celestial mechanics. To confirm such a possibility let us show that the clouds will not evaporate although they are heated by soft $\mathrm{x}$-ray emission from the intergalactic gas. On the other hand, the clouds will not collapse under the action of the external pressure ( $\mathrm{P}_{\text {ext }}$ ) and form stars during their flight to the E galaxy. The fulfillment of these statements substantially depends on the parameters of the cloud and of the intergalactic medium. Time scales of the 
processes in question are smaller than the time of the cloud flight to E galaxy (Sotnikova 1988a).

Taking the cloud to be initially homogeneous both in temperature $\left(\mathrm{T}_{\mathrm{Cl}}=\mathrm{T}_{\mathrm{Cl}}(t)\right)$ and in density $\left(\rho_{\mathrm{C} \ell}=\rho_{\mathrm{C} \ell}(t)\right)$, one may write down the equations determining the changes of cloud radius $R(t)$ and its temperature (Sotnikova 1986).

$$
\begin{aligned}
& \frac{3}{5} M R \frac{d^{2} R}{d t^{2}}=\frac{3 M k T_{C \ell}}{\mu m_{H}}-4 \pi R^{3} P_{\text {ext }}-\frac{3}{5} \frac{G M^{2}}{R}, \\
& \frac{1}{2 R^{2}} \frac{d}{d t}\left(\frac{3 M k T_{C \ell}}{\mu m_{H}} R^{2}\right)=\int_{V(t)}(\Gamma-\Lambda) d V, V(t)=\frac{4 \pi}{3} R^{3}(t), \\
& T_{C \ell}=T_{O}, R=R_{O} t=0 .
\end{aligned}
$$

Energy gain due to heating by $x$-rays has the following form: $\Gamma=3 \delta \rho_{x} c / 2 R$ (Sotnikova 1986), where $\rho_{x}$ is the energy density and $\delta$ is the rate of transformation of the energy of $x-$ rays to thermal energy. In calculating the cooling function $\Lambda$ several different abundances of heavy elements were considered. Numerical solution of simultaneous equations (1) has shown that the cloud neither evaporates nor collapses if its mass is: $30 M_{\odot}<M<200 M_{\mathcal{O}^{\prime}}$, if the gas is deficient in heavy elements, and the ratio $\mathrm{P}_{\mathrm{ext}} / \mathrm{n}_{\mathrm{cl}} \mathrm{kT}_{\mathrm{cl}}$ is not too large. Therefore, one can consider the gas flow as a stream consisting of discrete particles.

The changes of the radius and temperature of the cloud having $M=50 M_{0}$ are shown in Figure 1 . 


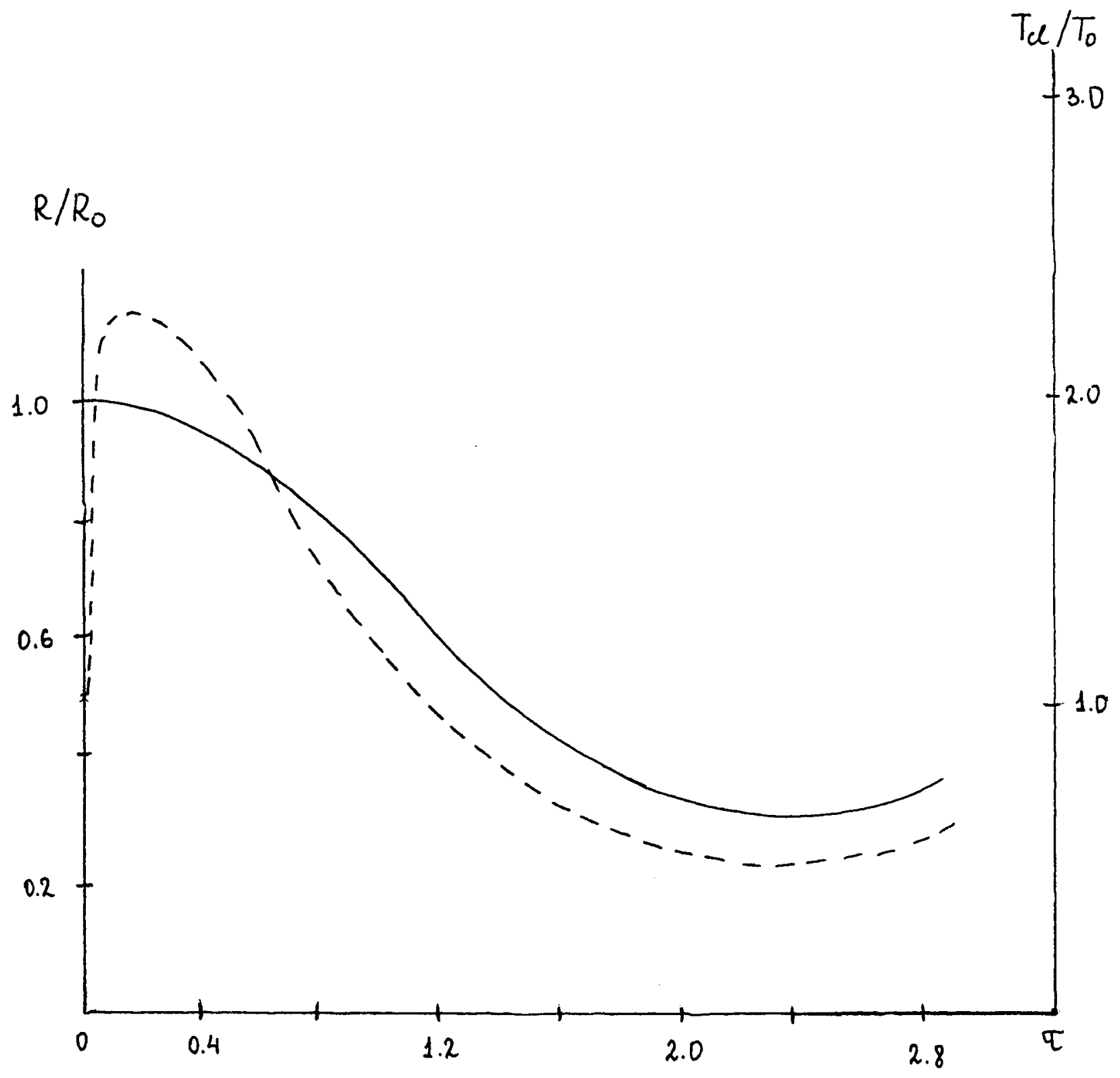

Figure 1. Changes of radius and temperature of a cloud by action of external pressure. $\mathrm{P}_{\text {ext }} / \mathrm{n}_{\mathrm{Cl}} \mathrm{kT}_{\mathrm{Cl}}=3, \mathrm{~T}_{\mathrm{O}}=50 \mathrm{~K}, \mathrm{R}_{\mathrm{O}}=2.5 \mathrm{kpc}$, $\delta=0.7, n_{C+} / n_{H}=0.1\left(n_{c} / n_{H}\right)_{0}^{\prime} n_{O} / n_{H}=0.5\left(n_{O} / n_{H}\right)_{O}^{\prime} \tau=t / 1.7 \times 10^{6}$ years. 
TRANSFER OF GAS TO AN E GALAXY IN A BINARY SYSTEM: NUMERICAL SIMULATION

As is known, orbits of galaxies in pairs are almost circular (Karachentsev 1987). Therefore one has to solve the circular restricted three-body problem for clouds ("particles") to simulate the gaseous stream. Because the gravitational potential of galaxies differs from that of a point mass, a modification of this problem must be made (Sotnikova 1988a). The stellar mass distribution in the disk containing clouds was taken to obtain a flat rotation curve. The disk plane coincides with the orbital one. The halo potential is supposed to be spherically symmetric.

The efficiency of gas transfer depends on the following main factors (for given $q=M_{E} / M_{S}$ ):

a) the ratio of the radius of the spiral galaxy $R_{s}$ to the distance between components $\mathrm{r}_{\mathrm{SE}}$;

b) spiral galaxy orientation relative to orbital momentum;

c) gas content in the spiral.

The value of $q$ may be estimated using the ratio of the luminosities $\mathrm{L}_{E} / \mathrm{L}_{\mathrm{S}}$. Usually $0.1<\mathrm{q}<10$ and the average value of $q$ is 1 - 2 (Karachentsev 1987).

A reasonable value of $R_{S} / r_{S E}$ may be estimated as follows. The outflow of gas from $S$ galaxy cannot occur if the initial rotation velocity $v_{0}$ of a cloud at the distance from the center of spiral galaxy equal to its radius $R_{S}$ is not large enough to reach the Lagrangian point $L_{1}$. Let $R_{S m i n}$ be the minimal value of $R_{S}$ for which $V_{0}$ satisfies this requirement. The value of $R_{s m i n} / \mu_{S E}$ and the sizes 
of critical Roche surfaces $R_{C r}$, min for point masses are given in Table 1 .

Table 1 .

\begin{tabular}{cccccc}
\hline $\mathrm{q}$ & $\mathrm{R}_{\mathrm{Smin}} / \mathrm{r}_{\mathrm{SE}}$ & $\mathrm{R}_{\mathrm{Cr}} \mathrm{min}^{/ \mathrm{r}_{\mathrm{SE}}}$ & $\mathrm{q}$ & $\mathrm{R}_{\mathrm{Smin}} / \mathrm{r}_{\mathrm{SE}}$ & $\mathrm{R}_{\mathrm{Cr}} \mathrm{min}^{/ \mathrm{r}_{\mathrm{SE}}}$ \\
\hline 0.1 & 0.421 & 0.594 & 2.0 & 0.198 & 0.313 \\
0.5 & 0.287 & 0.439 & 5.0 & 0.153 & 0.242 \\
1.0 & 0.240 & 0.373 & 10.0 & 0.125 & 0.197 \\
\hline
\end{tabular}

For outflow of gas from the $S$ galaxy to be possible the following inequality must be satisfied:

$$
\mathrm{R}_{\mathrm{S}} / \mathrm{r}_{\mathrm{SE}}>\mathrm{R}_{\mathrm{Smin}} / \mathrm{r}_{\mathrm{SE}}
$$

Taking into account the mean value of $\langle q\rangle * 2$ calculated from the data of (Karachentsev 1987, Faber and Gallagher 1979), one can see from Table 1 that $R_{\mathrm{Smin}} / \mathrm{r}_{\mathrm{SE}} \simeq 0.20$.

Further, one can find the mean values of the parameters of $S$ galaxies in pairs with and without observational traces of interaction and compare them with the value of $\mathrm{R}_{\mathrm{Smin}} / \mathrm{r}_{\mathrm{SE}}$.

In a sample of 41 pairs without any visible interaction $\left\langle\mathrm{R}_{\mathrm{S}}\right\rangle /\left\langle\mathrm{r}_{\mathrm{SE}}\right\rangle=0.17$; i.e., $\left\langle\mathrm{R}_{\mathrm{Smin}} / \mathrm{r}_{\mathrm{SE}}\right.$. Thus the "mean" galaxy of the sample does not satisfy the requirement for gas flow (2).

The $S$ galaxies from a sample of 38 pairs with observational traces of tidal interaction given in Karachentsev (1987) have $\left\langle\mathrm{R}_{\mathrm{S}}\right\rangle /\left\langle\mathrm{r}_{\mathrm{SE}}\right\rangle=0.40$ and therefore in these systems transfer of gas to E galaxy is possible. 
To get the characteristics of the gaseous stream from $\mathrm{S}$ galaxy numerical solution of equations of test particle motion were carried out (Sotnikova 1988a). Many sets of initial conditions were used and two different cases were considered.

(i) The direction of spiral galaxy spin and that of orbital momentum are the same.

\section{(ii) These directions are opposite.}

The main results of the computations are:

1. In the case (i) the time scale of stream formation is much smaller than in case ( $i$ i). For example, in the last case the test particle has stayed on its circular orbit in s galaxy during the period of revolution of the whole system $\left(\sim 3.10^{8}\right.$ years $)$.

2. In case ( $i$ ) during one revolution of the binary system the stream evolves to quasi steady state (Figure 2).

The S galaxy loses about 0.58 of the total amount of gas per period of the disk revolution $\left(\sim 10^{8}\right.$ years). Mean values of the parameters for systems with interacting S-E galaxies from Karachentsev's list (1987) are: $r_{\mathrm{SE}}=34 \mathrm{kpc}, \mathrm{M}_{\mathrm{S}}=(2-3)$ - $10^{11_{M_{0}}}$. The corresponding cloud transfer rate is $M_{\text {gas }} \cong$ $(0.1-0.3) M_{0} y^{-1}$ provided the total mass of gas in the galaxy is about $0.1 \mathrm{M}_{\text {stars }}$. 


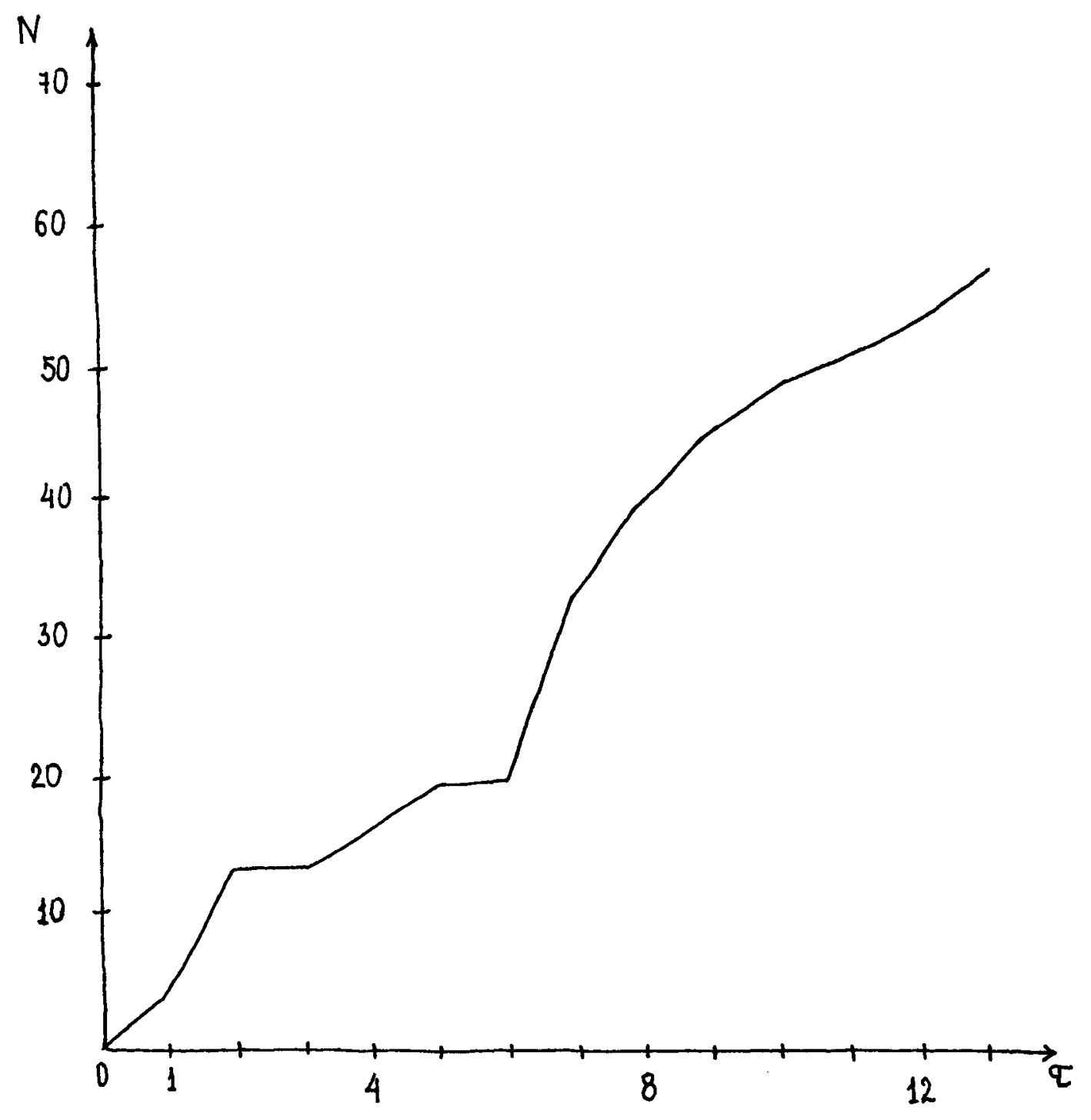

Figure 2. The number of test particles $N$ (the units are arbitrary) lost by spiral galaxy at the time $\tau=t / 2.1 \cdot 10^{8}$ years $\left(r_{S E}=34 \mathrm{kpc}, \mathrm{M}_{\mathrm{S}}=2 \cdot 10^{11} \mathrm{M}_{\mathrm{O}}\right), \mathrm{q}=\mathrm{I}, \mathrm{R}_{\mathrm{S}} / \mathrm{r}_{\mathrm{SE}}=0.35$, $\mathrm{R}_{\mathrm{E}} / \mathrm{r}_{\mathrm{SE}}=0.35$. 


\section{CONCLUSIONS}

In the course of $S-E$ pair evolution $\left(t_{e v} \sim 10^{9}\right.$ years) a significant amount of gas may be transferred to $E$ galaxy $\left(\dot{M}_{\text {gas }} \cdot t_{\mathrm{ev}} \simeq 10^{8} \mathrm{M}_{\mathrm{o}}\right)$. This value is of the order of observed HI content in $\mathrm{E}$ galaxies which are the members of multiple systems.

One may mention also that the presence of emission lines in spectra of many E components of close S-E pairs (258) may be caused by gas gained by E galaxies due to the tidal interaction with S galaxies.

\section{REFERENCES}

Bottinelli, L., and Gougenheim, L. 1978, Astron. Astrophys., 76, 176.

Demin, V. V., Zasov, A. V., Dibaj, Eh. A., and Tomov, A. N. 1984, Astron. Zh., 61, 625 .

Faber, S. M., Gallagher, J. S. 1979, Annu. Rev. Astron. Astrophys., 17, 135 .

van Gorkom, J. H., Knapp, G. R., Raimond, E., Faber, S. M., Gallagher, J. S. 1986, Astron. J., 91, 791.

Karachentsev, I. D. 1987, Dvojnye Galaktiki, Nauka, Moscow, USSR, $248 \mathrm{pp}$.

Smirnov, M. A., and Komberg, B. V. 1980, Astrofizika, 16, 431 . Sotnikova, N. Ya. 1986, Astrifizika, 25, 139.

Sotnikova, N. Ya. 1988a, Astrofizika, 28, 495. 
Sotnikova, N. Ya. 1988b, Ph.D. Thesis, Leningrad University, Leningrad.

Varnas, S. R., Bertola, F., Galleta, G. 1987, Astrophys. J.,. 313,69 